\title{
Dynamic Analysis of a Shallow Buried Tunnel Influenced by a Neighboring Semi-cylindrical Hill and Semi-cylindrical Canyon
}

\author{
Xiaotang $\mathrm{Lv}^{1 *}$, Cuiling Ma${ }^{1}$, Meixiang Fang ${ }^{1}$ \\ 1 Department of Civil Engineering, Hefei University \\ No.99 Jinxiu Street, Hefei, 230061, China \\ *Corresponding author, e-mail: Ivxiaotang@sina.com
}

Received: 10 April 2019, Accepted: 24 June 2019, Published online: 06 August 2019

\begin{abstract}
This paper provides a dynamic analysis of the response of a subsurface cylindrical tunnel to SH waves influenced by a neighboring semi-cylindrical hill and semi-cylindrical canyon in half-space using complex functions. For convenience in finding a solution, the halfspace is divided into two parts and the scattered wave functions are constructed in both parts. Then the mixed boundary conditions are satisfied by moving coordinates. Finally, the problem is reduced to solving a set of infinite linear algebraic equations, for which the unknown coefficients are obtained by truncation of the infinite set of equations. The effects of the incident angles and frequencies of $\mathrm{SH}$ waves, as well as of the radius of the tunnel, hill, and canyon on the dynamic stress concentration of the tunnel are studied. The results show that the hill and canyon have a significant effect on the dynamic stress concentration of the tunnel.
\end{abstract}

\section{Keywords}

SH waves, dynamic stress concentration, tunnel, semi-cylindrical hill, semi-cylindrical canyon

\section{Introduction}

Taking the scattering of elastic waves and the concentration of dynamic stress as the theoretical background, seismic analysis and dynamic analysis of underground structures are important topics in seismic engineering research. According to the research objects, the research on the dynamic characteristics of underground structures under SH waves has fallen generally into two categories: the anti-plane motion of inclusions, holes, and linings in halfspace, and the dynamic response of underground structures that are influenced by surface topographies (canyons, alluvial valleys, hills, and so on). Methods of solution are mainly analytical methods and numerical methods. Mathematically, the diffraction of elastic waves is solved by a set of wave motion equations and prescribed boundary conditions. The wave function expansion method is widely used for solving boundary value problems analytically. In 1979 [1] and 1984 [2], this method was used to analyze the scattering of SH waves by a circular tunnel and by twin circular tunnels in an elastic half-space. Also, for the scattering of SH waves by other structures in half-space, many meaningful results have been obtained by wave function expansion method [3-7]. Using complex function, Liu et al. [8] provided a new analytical method for two-dimensional dynamic stress concentration problems. In 1988, the complex function method was further developed and applied to the problem of dynamic stress concentration in the neighborhood of a circular hole in anisotropic media [9]. Based on the theory of complex functions, the scattering of SH waves by a shallow-embedded lining structure [10], a subsurface cylindrical cavity [11] and a cavity of arbitrary shape in half-space [12] was studied. In addition, numerical methods, such as the direct boundary element method [13], finite element method [14], and indirect boundary element method [15] are effective methods for studying the scattering of elastic waves.

Compared with studies of underground structures in half-space, research results on the interaction between surface and subsurface topographies are relatively few. For the scattering problems of SH waves, in 1999, Lee et al. [16] analyzed the diffraction by a surface semicircular canyon on top of an underground circular unlined tunnel (cavity) in a homogeneous elastic half-space. This analysis was extended to study the diffraction caused by a semi-circular rigid foundation with an underground rigid circular tunnel directly below it [17]. In addition, a closed-form analytic solution was presented in 2004 [18] for scattering by 
a semi-circular cylindrical hill with a semi-circular concentric tunnel inside on a half-space. Using complex function, Liu and Wang [19] studied the scatting of SH waves by a semi-cylindrical hill above a subsurface cavity in halfspace and presented computational results of surface displacement. Based on the same method, Lv [20] solved the interaction between multiple semi-cylindrical hills and a subsurface elastic cylindrical inclusion under SH waves and provided the displacement variation of the hill's surface. In 2016, an analytic solution for the scattering of antiplane SH waves by a shallow semi-elliptical hill with a concentric elliptical tunnel was presented [21]. Using the direct boundary element method, the seismic response of semisine-shaped canyons above a subterranean cavity (hole) of different dimensions, depths, and locations was examined under vertically incident SV and P waves [22].

In a half-space containing a semi-cylindrical hill connected to a semi-cylindrical canyon, this paper analyzes the dynamic stress concentrations of a shallow buried cylindrical tunnel under $\mathrm{SH}$ waves. Based on the wave function expansion method, complex functions and moving coordinates system are used in different solution regions to construct wave functions and to satisfy mixed boundary conditions. Finally, the solution is reduced to solving a set of infinite linear algebraic equations. The numerical results of dynamic stress concentration factors are obtained by truncation of the infinite equations.

\section{Calculational model}

The displacement induced by SH waves in linear, homogeneous, isotropic media is normal to the xoy-plane, and the corresponding stresses exist only in the xoy-plane. Therefore, in an elastic half-space with a semi-cylindrical hill connected to a semi-cylindrical canyon, the calculational model of a shallow buried cylindrical tunnel under SH wave can be simplified to a 2D model, as shown Fig. 1. $\mathrm{O}_{1}, \mathrm{O}_{2}$ and $\mathrm{O}_{3}$ represent the centers of the semi-cylindrical hill, the semi-cylindrical canyon and the cylindrical tunnel, respectively, and $R_{1}, R_{2}$ and $R_{3}$ denote their respective radii. $\mathrm{S}$ is the horizontal surface of the half-space. Boundaries of the semi-cylindrical hill, the semi-cylindrical canyon and the cylindrical tunnel are $\mathrm{C}, \overline{\mathrm{S}}_{2}$ and $\mathrm{H}$, respectively.

Under SH waves, solving the dynamic stress concentration of the cylindrical tunnel in the calculational model means, solving the governing equations of $\mathrm{SH}$ waves that satisfy stress free boundary conditions on the boundaries of the semi-cylindrical hill $\mathrm{C}$, the semi-cylindrical canyon $\overline{\mathrm{S}}_{2}$

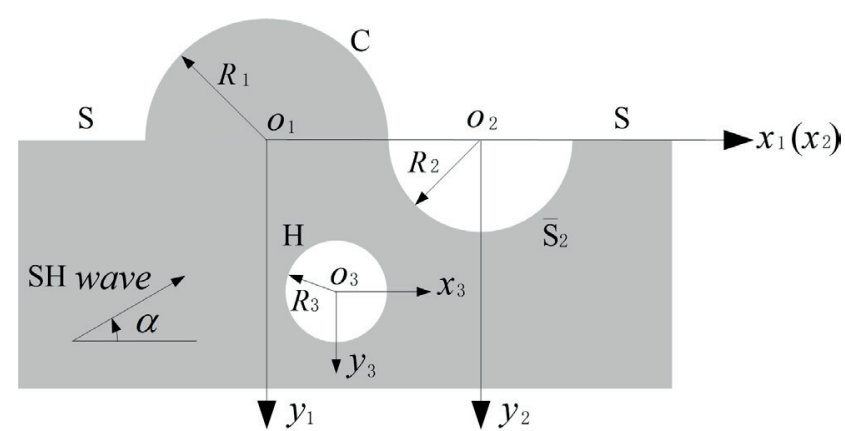

Fig. 1 Calculational model

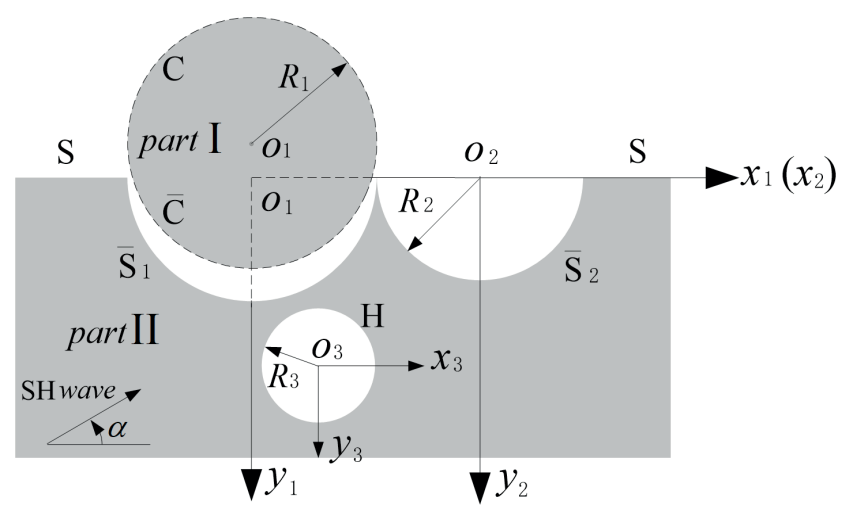

Fig. 2 Two solution regions

and the cylindrical tunnel $\mathrm{H}$. To achieve the solution, the calculational model shown in Fig. 1 is divided into two regions, as shown in Fig. 2. The first one is a circular area including the boundary and the hill's boundary $\mathrm{C}$. The second area contains all the remaining parts, including the horizontal surface $\mathrm{S}$, the cylindrical tunnel $\mathrm{H}$, the semi-cylindrical canyon $\overline{\mathrm{S}}_{2}$ and the boundary $\overline{\mathrm{S}}_{1} \cdot \overline{\mathrm{S}}_{1}$ and $\overline{\mathrm{C}}$ are common boundaries of the two regions.

With the points $o_{1}, o_{2}$ and $o_{3}$ as coordinate origins, three rectangular coordinate systems $x_{1}-O_{1}-y_{1}, x_{2}-O_{2}-y_{2}$ and $x_{3}-O_{3}-y_{3}$ are established, corresponding to three complex planes $\left(z_{1}, \bar{z}_{1}\right),\left(z_{2}, \bar{z}_{2}\right)$, and $\left(z_{3}, \bar{z}_{3}\right)$.

\section{Solution}

\subsection{Basic equations}

Introducing complex variables $z=x+\mathrm{i} y, z=x-\mathrm{i} y$, the form of the Helmholtz equation in the complex plane $(z, \bar{z})$ is as Eq. (1):

$$
\frac{\partial^{2} W}{\partial z \partial \bar{z}}+\frac{1}{4} k^{2} W=0
$$

In a polar coordinate system, the corresponding stresses can be expressed as Eq. (2):

$$
\tau_{r z}=\mu\left(\frac{\partial W}{\partial z} e^{i \theta}+\frac{\partial W}{\partial \bar{z}} e^{-\mathrm{i} \theta}\right), \tau_{\theta z}=\mathrm{i} \mu\left(\frac{\partial W}{\partial z} e^{\mathrm{i} \theta}-\frac{\partial W}{\partial \bar{z}} e^{-\mathrm{i} \theta}\right) .
$$


Here $W$ stands for the displacement function, the time dependence of $W$ is $e^{-\mathrm{i} \omega \theta}$ (this factor will be omitted in the following discussion). $k=\omega / c_{s}$, where $\omega$ is the circular frequency, and $c_{s}$ and $\mu$ are the shear wave velocity and the mass density of medium respectively.

\subsection{Incident wave and reflected wave}

In the complex plane $\left(z_{1}, \bar{z}_{1}\right)$, the incident wave $W^{(i)}$, the reflected wave $W^{(r)}$, and the corresponding stresses are Eqs. (3)-(6):

$$
\begin{aligned}
& W_{\left(z_{1}, \bar{z}_{1}\right)}^{(i)}=W_{0} e^{\frac{\mathrm{i} k}{2}\left[z_{1} e^{\mathrm{i} \alpha}+\bar{z}_{1} e^{-\mathrm{i} \alpha}\right]}, \\
& W_{\left(\bar{z}_{1}, \bar{z}_{1}\right)}^{(r)}=W_{0} e^{\frac{\mathrm{i} k}{2}\left[z_{1} e^{-\mathrm{i} \alpha}+\bar{z}_{1} e^{\mathrm{i} \alpha}\right]}, \\
& \tau_{r z_{1}}^{(i)}=\mathrm{i} \mu k W_{0} \cos \left(\theta_{1}+\alpha\right) e^{\mathrm{i} k\left|z_{1}\right| \cos \left(\theta_{1}+\alpha\right)}, \\
& \tau_{r z_{1}}^{(r)}=\mathrm{i} \mu k W_{0} \cos \left(\theta_{1}-\alpha\right) e^{\mathrm{i} k\left|z_{1}\right| \cos \left(\theta_{1}-\alpha\right)} .
\end{aligned}
$$

In the complex plane $\left(z_{2}, \bar{z}_{2}\right)$, Eqs. (3)-(6) take the forms as Eqs. (7)-(10):

$$
\begin{aligned}
& W_{\left(z_{2}, \bar{z}_{2}\right)}^{(i)}=W_{0} e^{\frac{\mathrm{i} k}{2}\left[\left(z_{2}-d^{\prime}\right) e^{\mathrm{i} \alpha}+\left(\bar{z}_{2}-\bar{d}^{\prime}\right) e^{-\mathrm{i} \alpha}\right]}, \\
& W_{\left(z_{2}, \bar{z}_{2}\right)}^{(r)}=W_{0} e^{\frac{\mathrm{i} k}{2}\left[\left(z_{2}-d^{\prime}\right) e^{-\mathrm{i} \alpha}+\left(\bar{z}_{2}-\bar{d}^{\prime}\right) e^{\mathrm{i} \alpha}\right]}, \\
& \tau_{r z_{2}}^{(i)}=\mathrm{i} \mu k W_{0} \cos \left(\theta_{2}+\alpha\right) e^{\frac{\mathrm{i} k}{2}\left[\left(z_{2}-d^{\prime}\right) e^{\mathrm{i} \alpha}+\left(\bar{z}_{2}-\bar{d}^{\prime}\right) e^{-\mathrm{i} \alpha}\right]}, \\
& \tau_{r z_{2}}^{(r)}=\mathrm{i} \mu k W_{0} \cos \left(\theta_{2}-\alpha\right) e^{\frac{\mathrm{i} k}{2}\left[\left(z_{2}-d^{\prime}\right) e^{-\mathrm{i} \alpha}+\left(\bar{z}_{2}-\bar{d}^{\prime}\right) e^{\mathrm{i} \alpha}\right]} .
\end{aligned}
$$

In the complex plane $\left(z_{3}, \bar{z}_{3}\right)$, Eqs. (3)-(6) are Eqs. (11)-(14):

$$
\begin{aligned}
& W_{\left(z_{3}, \bar{z}_{3}\right)}^{(i)}=W_{0} e^{\frac{\mathrm{i} k}{2}\left[\left(z_{3}+h\right) e^{\mathrm{i} \alpha}+\left(\overline{z_{3}}+\bar{h}\right) e^{-\mathrm{i} \alpha}\right]} \\
& W_{\left(z_{3}, \bar{z}_{3}\right)}^{(r)}=W_{0} e^{\frac{\mathrm{i} k}{2}\left[\left(z_{3}+h\right) e^{-\mathrm{i} \alpha}+\left(\overline{z_{3}}+\bar{h}\right) e^{\mathrm{i} \alpha}\right]} \\
& \tau_{r z_{3}}^{(i)}=\mathrm{i} \mu k W_{0} \cos \left(\theta_{3}+\alpha\right) e^{\frac{\mathrm{i} k\left[\left(z_{3}+h\right) e^{\mathrm{i} \alpha}+\left(\overline{z_{3}}+\bar{h}\right) e^{-\mathrm{i} \alpha}\right]}{2}} \\
& \tau_{r z_{3}}^{(r)}=\mathrm{i} \mu k W_{0} \cos \left(\theta_{3}-\alpha\right) e^{\frac{\mathrm{i} k}{2}\left[\left(z_{3}+h\right) e^{-\mathrm{i} \alpha}+\left(\bar{z}_{3}+\bar{h}\right) e^{\mathrm{i} \alpha}\right]}
\end{aligned}
$$

\subsection{Standing wave in circular region}

A standing wave $W^{(s t)}$ will appear in the circular region under the disturbance of SH waves, and the corresponding stress function should satisfy the boundary conditions of being free on the upper half boundary $\mathrm{C}$ and being continuous on the lower half boundary $\overline{\mathrm{C}}$. In the complex plane $\left(z_{1}, \bar{z}_{1}\right)$, displacement and stress solutions satisfying these conditions take the forms as Eqs. (15-16):

$$
\begin{aligned}
& W_{\left(z_{1}, \bar{z}_{1}\right)}^{(s t)}=W_{0} \sum_{n=-\infty}^{\infty} \sum_{m=-\infty}^{\infty} C_{m} \frac{J_{m-1}\left(k R_{1}\right)-J_{m+1}\left(k R_{1}\right)}{J_{n-1}\left(k R_{1}\right)-J_{n+1}\left(k R_{1}\right)} a_{m n} J_{n}\left(k\left|z_{1}\right|\right)\left[\frac{z_{1}}{\left|z_{1}\right|}\right]^{n}, \\
& \tau_{r z_{1}}^{(s t)}=\frac{\mu k W_{0}}{2} \sum_{n=-\infty}^{+\infty} \sum_{m=-\infty}^{+\infty} C_{m} \frac{J_{m-1}\left(k R_{1}\right)-J_{m+1}\left(k R_{1}\right)}{J_{n-1}\left(k R_{1}\right)-J_{n+1}\left(k R_{1}\right)} a_{m n} \\
& \times\left[J_{n-1}\left(k\left|z_{1}\right|\right)-J_{n+1}\left(k\left|z_{1}\right|\right)\right]\left[\frac{z_{1}}{\left|z_{1}\right|}\right]^{n} .
\end{aligned}
$$

The expression of $a_{m n}$ can be found in [20]. $C_{m}$ are undetermined coefficients and $W_{0}$ is the maximum displacement amplitude of the standing wave.

\subsection{Scattered wave}

With the incidence of SH waves, the total scattered wave field $W_{\text {II }}^{(s)}$ in part II (the second area) can be expressed as Eq. (17):

$$
W_{\mathrm{II}}^{(s)}=W_{\bar{S}_{1}}^{(s)}+W_{\bar{S}_{2}}^{(s)}+W_{H}^{(s)} .
$$

Here, $W_{\bar{S}_{1}}^{(s)}$ and $W_{\bar{S}_{2}}^{(s)}$ are scattered waves caused by $\overline{\mathrm{S}}_{1}$ and $\overline{\mathrm{S}}_{2} ; W_{H}^{(s)}$ is the scattered wave due to the existence of the tunnel $H$.

The total scattered wave field $W_{\text {II }}^{(s)}$ should satisfy stress free conditions on the horizontal surface of the half-space. According to the symmetry of SH wave scattering and the multi-polar coordinates, the scattered waves satisfying the above conditions can be constructed. In the complex plane $\left(z_{1}, \bar{z}_{1}\right)$, they take the forms as Eqs. (18)-(20):

$$
\begin{aligned}
& W_{\bar{S}_{1},\left(z_{1}, \bar{z}_{1}\right)}^{(s)}=W_{0} \sum_{m=0}^{\infty} A_{m} H_{m}^{(1)}\left(k\left|z_{1}\right|\right)\left\{\left[\frac{z_{1}}{\left|z_{1}\right|}\right]^{m}+\left[\frac{z_{1}}{\left|z_{1}\right|}\right]^{-m}\right\}, \\
& W_{\bar{S}_{2,\left(z_{1}, \bar{z}_{1}\right)}^{(s)}}^{(-m}=W_{0} \sum_{m=0}^{\infty} B_{m} H_{m}^{(1)}\left(k\left|z_{1}-d\right|\right)\left\{\left[\frac{z_{1}-d}{\left|z_{1}-d\right|}\right]^{m}+\left[\frac{z_{1}-d}{\left|z_{1}-d\right|}\right]^{-m}\right\},
\end{aligned}
$$

in which, $d$ is the complex coordinate of the point $O_{2}$, the center of the semi-cylindrical canyon $\overline{\mathrm{S}}_{2}$ in the complex plane $\left(z_{1}, \bar{z}_{1}\right)$, and $A_{m}$ and $B_{m}$ are undetermined coefficients.

$$
\begin{aligned}
& W_{H,\left(z_{1}, \bar{z}_{1}\right)}^{(s)}=W_{0} \sum_{m=-\infty}^{\infty} D_{m}\left\{H_{m}^{(1)}\left(k\left|z_{1}-h\right|\right)\left[\frac{z_{1}-h}{\left|z_{1}-h\right|}\right]^{m}\right. \\
& \left.+H_{m}^{(1)}\left(k\left|z_{1}-\bar{h}\right|\right)\left[\frac{z_{1}-\bar{h}}{\left|z_{1}-\bar{h}\right|}\right]^{-m}\right\} .
\end{aligned}
$$


Here $h$ is the complex coordinate of the cylindrical tunnel's center $O_{3}$ in the complex plane $\left(z_{1}, \bar{z}_{1}\right)$, and $\bar{h}$ is its complex conjugate; $D_{m}$ are undetermined coefficients.

The corresponding stresses are as Eqs. (21)-(23):

$$
\begin{aligned}
& \tau_{r z_{1}, \bar{S}_{1}}^{(s)}=\frac{\mu k W_{0}}{2} \sum_{m=0}^{\infty} A_{m}\left[H_{m-1}^{(1)}\left(k\left|z_{1}\right|\right)-H_{m+1}^{(1)}\left(k\left|z_{1}\right|\right)\right] \\
& \times\left\{\left[\frac{z_{1}}{\left|z_{1}\right|}\right]^{m}+\left[\frac{z_{1}}{\left|z_{1}\right|}\right]^{-m}\right\}, \\
& \tau_{r z_{1}, \bar{S}_{2}}^{(s)}=\frac{\mu k W_{0}}{2} \sum_{m=0}^{\infty} B_{m}\left(\left[\begin{array}{l}
H_{m-1}^{(1)}\left(k\left|z_{1}-d\right|\left[\frac{z_{1}-d}{\left|z_{1}-d\right|}\right]^{m-1}\right. \\
-H_{m+1}^{(1)}\left(k\left|z_{1}-d\right|\right)\left[\frac{z_{1}-d}{\left|z_{1}-d\right|}\right]^{-(m+1)}
\end{array}\right] e^{i \theta_{1}}\right. \\
& \left.+\left[\begin{array}{l}
-H_{m+1}^{(1)}\left(k\left|z_{1}-d\right|\left[\frac{z_{1}-d}{\left|z_{1}-d\right|}\right]^{m+1}\right. \\
+H_{m-1}^{(1)}\left(k\left|z_{1}-d\right|\right)\left[\frac{z_{1}-d}{\left|z_{1}-d\right|}\right]^{-(m-1)}
\end{array}\right] e^{-\mathrm{i} \theta_{1}}\right\},
\end{aligned}
$$

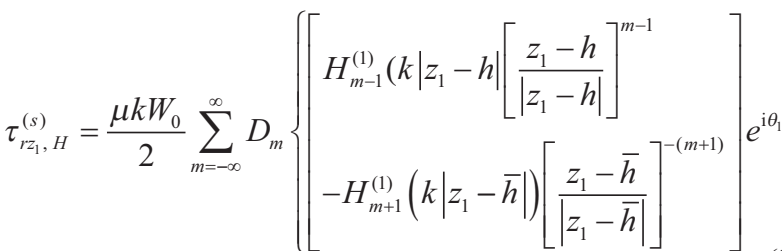

$$
\begin{aligned}
& \left.+\left[\begin{array}{l}
-H_{m+1}^{(1)}\left(k\left|z_{1}-h\right|\left[\frac{z_{1}-h}{\left|z_{1}-h\right|}\right]^{m+1}\right. \\
+H_{m-1}^{(1)}\left(k\left|z_{1}-\bar{h}\right|\right)\left[\frac{z_{1}-\bar{h}}{\left|z_{1}-\bar{h}\right|}\right]^{-(m-1)}
\end{array}\right] e^{-\mathrm{i} \theta_{1}}\right\} .
\end{aligned}
$$

By moving coordinates, Eqs. (18)-(23) in the complex plane $\left(z_{2}, \bar{z}_{2}\right)$ take the forms as Eqs. (24)-(29):

$$
\begin{aligned}
& W_{\bar{S}_{1},\left(z_{2}, \bar{z}_{2}\right)}^{(s)}=W_{0} \sum_{m=0}^{\infty} A_{m} H_{m}^{(1)}\left(k\left|z_{2}-d^{\prime}\right|\right)\left\{\left[\frac{z_{2}-d^{\prime}}{\left|z_{2}-d^{\prime}\right|}\right]^{m}+\left[\frac{z_{2}-d^{\prime}}{\left|z_{2}-d^{\prime}\right|}\right]^{-m}\right\}, \\
& W_{\bar{S}_{2},\left(z_{2}, \bar{z}_{2}\right)}^{(s)}=W_{0} \sum_{m=0}^{\infty} B_{m} H_{m}^{(1)}\left(k\left|z_{2}\right|\right)\left\{\left[\frac{z_{2}}{\left|z_{2}\right|}\right]^{m}+\left[\frac{z_{2}}{\left|z_{2}\right|}\right]^{-m}\right\}, \\
& W_{H,\left(z_{2}, \bar{z}_{2}\right)}^{(s)}=W_{0} \sum_{m=-\infty}^{\infty} D_{m}\left\{H_{m}^{(1)}\left(k\left|z_{2}-h^{\prime}\right|\right)\left[\frac{z_{2}-h^{\prime}}{\left|z_{2}-h^{\prime}\right|}\right]^{m}\right. \\
& +H_{m}^{(1)}\left(k\left|z_{2}-\overline{h^{\prime}}\right|\right)\left[\frac{z_{2}-\overline{h^{\prime}}}{\left.\left.\left|z_{2}-\overline{h^{\prime}}\right|\right]^{-m}\right\}}\right.
\end{aligned}
$$

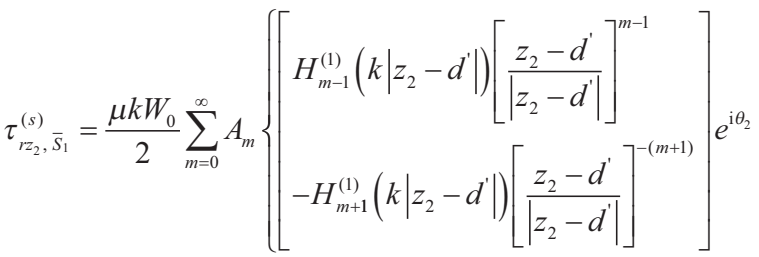

$$
\begin{aligned}
& \left.+\left[\begin{array}{l}
-H_{m+1}^{(1)}\left(k\left|z_{2}-d^{\prime}\right|\right)\left[\frac{z_{2}-d^{\prime}}{\left|z_{2}-d^{\prime}\right|}\right]^{m+1} \\
+H_{m-1}^{(1)}\left(k\left|z_{2}-d^{\prime}\right|\right)\left[\frac{z_{2}-d^{\prime}}{\left|z_{2}-d^{\prime}\right|}\right]^{-(m-1)}
\end{array}\right] e^{-\mathrm{i} \theta_{2}}\right\}, \\
& \tau_{r_{2}, \bar{S}_{2}}^{(s)}=\frac{\mu k W_{0}}{2} \sum_{m=0}^{\infty} B_{m}\left[H_{m-1}^{(1)}\left(k\left|z_{2}\right|\right)-H_{m+1}^{(1)}\left(k\left|z_{2}\right|\right)\right] \\
& \times\left\{\left[\frac{z_{2}}{\left|z_{2}\right|}\right]^{m}+\left[\frac{z_{2}}{\left|z_{2}\right|}\right]^{-m}\right\},
\end{aligned}
$$$$
\tau_{r_{2}, H}^{(s)}=\frac{\mu k W_{0}}{2} \sum_{m=-\infty}^{\infty} D_{m}\left\{\begin{array}{l}
H_{m-1}^{(1)}\left(k\left|z_{2}-h^{\prime}\right|\left[\frac{z_{2}-h^{\prime}}{\left|z_{2}-h^{\prime}\right|}\right]^{m-1}\right. \\
-H_{m+1}^{(1)}\left(k\left|z_{2}-\overline{h^{\prime}}\right|\right)\left[\frac{z_{2}-\overline{h^{\prime}}}{\left|z_{2}-\overline{h^{\prime}}\right|}\right]^{-(m+1)}
\end{array} e^{\mathrm{i} \theta_{2}}\right.
$$$$
\left.+\left[\begin{array}{l}
-H_{m+1}^{(1)}\left(k\left|z_{2}-h^{\prime}\right|\left[\frac{z_{2}-h^{\prime}}{\left|z_{2}-h^{\prime}\right|}\right]^{m+1}\right. \\
+H_{m-1}^{(1)}\left(k\left|z_{2}-\overline{h^{\prime}}\right|\right)\left[\frac{z_{2}-\overline{h^{\prime}}}{\left|z_{2}-\overline{h^{\prime}}\right|}\right]^{-(m-1)}
\end{array}\right] e^{-\mathrm{i} \theta_{2}}\right\} .
$$

In Eqs. (24)-(29), $h^{\prime}$ and $d^{\prime}$ are complex coordinates of the cylindrical tunnel's center $O_{3}$ and the semi-cylindrical hill's center $O_{1}$ in the complex plane $\left(z_{2}, \bar{z}_{2}\right), \bar{h}^{\prime}$ is the complex conjugate of $h^{\prime}$.

In the complex plane $\left(z_{3}, \bar{z}_{3}\right)$, Eqs. (18)-(23) can be written as Eqs. (30)-(35):

$$
\begin{aligned}
& W_{\left.\bar{S}_{1}, z_{3}, \bar{z}_{3}\right)}^{(s)}=W_{0} \sum_{m=0}^{\infty} A_{m} H_{m}^{(1)}\left(k\left|z_{3}+h\right|\right)\left\{\left[\frac{z_{3}+h}{\left|z_{3}+h\right|}\right]^{m}+\left[\frac{z_{3}+h}{\left|z_{3}+h\right|}\right]^{-m}\right\}, \\
& W_{S_{2},\left(z_{3}, \bar{z}_{3}\right)}^{(s)}=W_{0} \sum_{m=0}^{\infty} B_{m} H_{m}^{(1)}\left(k\left|z_{3}+h^{\prime}\right|\right)\left\{\left[\frac{z_{3}+h^{\prime}}{\left|z_{3}+h^{\prime}\right|}\right]^{m}+\left[\frac{z_{3}+h^{\prime}}{\left|z_{3}+h^{\prime}\right|}\right]^{-m}\right\}, \\
& W_{H,\left(z_{3}, \bar{z}_{3}\right)}^{(s)}=W_{0} \sum_{m=-\infty}^{\infty} D_{m}\left\{\begin{array}{l}
H_{m}^{(1)}\left(k\left|z_{3}\right|\right)\left[\frac{z_{3}}{\left|z_{3}\right|}\right]^{m} \\
\left.+H_{m}^{(1)}\left(k\left|z_{3}-\bar{h}+h\right|\right)\left[\frac{z_{3}-\bar{h}+h}{\left|z_{3}-\bar{h}+h\right|}\right]^{-m}\right\},
\end{array}\right.
\end{aligned}
$$




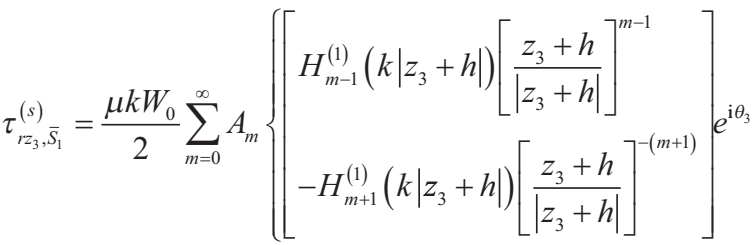

$$
\begin{aligned}
& \left.+\left[\begin{array}{l}
-H_{m+1}^{(1)}\left(k\left|z_{3}+h\right|\right)\left[\frac{z_{3}+h}{\left|z_{3}+h\right|}\right]^{m+1} \\
+H_{m-1}^{(1)}\left(k\left|z_{3}+h\right|\right)\left[\frac{z_{3}+h}{\left|z_{3}+h\right|}\right]^{-(m-1)}
\end{array}\right] e^{-\mathrm{i} \theta_{3}}\right\},
\end{aligned}
$$

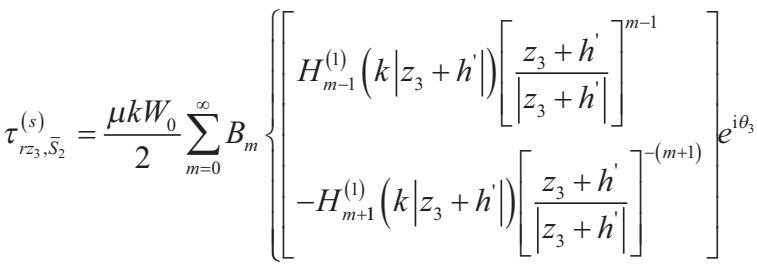

$$
\begin{aligned}
& +\left[\begin{array}{l}
-H_{m+1}^{(1)}\left(k \mid z_{3}+h^{\prime}\right)\left[\frac{z_{3}+h^{\prime}}{\left|z_{3}+h^{\prime}\right|}\right]^{m+1} \\
+H_{m-1}^{(1)}\left(k\left|z_{3}+h^{\prime}\right|\right)\left[\frac{z_{3}+h^{\prime}}{\left|z_{3}+h^{\prime}\right|}\right]^{-(m-1)}
\end{array}\right] e^{-\mathrm{i} \theta_{3}},,
\end{aligned}
$$

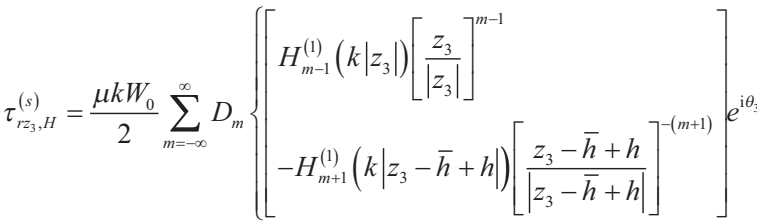

$$
\begin{aligned}
& \left.+\left[\begin{array}{l}
-H_{m+1}^{(1)}\left(k\left|z_{3}\right|\right)\left[\frac{z_{3}}{\left|z_{3}\right|}\right]^{m+1} \\
+H_{m-1}^{(1)}\left(k\left|z_{3}-\bar{h}+h\right|\right)\left[\frac{z_{3}-\bar{h}+h}{\left|z_{3}-\bar{h}+h\right|}\right]^{-(m-1)}
\end{array}\right] e^{-i \theta_{3}}\right\} .
\end{aligned}
$$

To conserve space, only the expressions of $\tau_{r z}$ are given in three coordinate systems. According to Eq. (2), the expressions of $\tau_{\theta z}$ can then be obtained.

\subsection{Boundary conditions and solving equations}

For the calculational model shown in Fig. 2, the displacement field and the stress field in two regions should satisfy four boundary conditions on three different boundaries. On the common boundaries $\overline{\mathrm{S}}_{1}$ and $\overline{\mathrm{C}}$, the conditions of stress and displacement continuous should be satisfied in the complex plane $\left(z_{1}, \bar{z}_{1}\right)$; and the stress free conditions on boundaries $\overline{\mathrm{S}}_{2}$ and $H$ should be satisfied in the complex planes $\left(z_{2}, \bar{z}_{2}\right)$ and $\left(z_{3}, \bar{z}_{3}\right)$ respectively. Therefore, the boundary conditions of the calculational model can be expressed as Eq. (36):

$$
\left\{\begin{array}{ll}
W_{\left(z_{1}, \bar{z}_{1}\right)}^{(s t)}=W_{\left(z_{1}, \bar{z}_{1}\right)}^{(i)}+W_{\left(z_{1}, \bar{z}_{1}\right)}^{(r)}+W_{\left.\bar{S}_{1}, \bar{z}_{1}, \bar{z}_{1}\right)}^{(s)}+W_{\bar{S}_{2},\left(z_{1}, \bar{z}_{1}\right)}^{(s)}+W_{H,\left(z_{1}, \bar{z}_{1}\right)}^{(s)} & \text { on } \overline{\mathrm{S}}_{1} \\
\tau_{r z_{1}}^{(s t)}=\tau_{r z_{1}}^{(i)}+\tau_{r z_{1}}^{(r)}+\tau_{r z_{1}, \bar{S}_{1}}^{(s)}+\tau_{r r_{1}, \bar{S}_{2}}^{(s)}+\tau_{r z_{1}, H}^{(s)} & \text { on } \overline{\mathrm{S}}_{1} \\
\tau_{r z_{2}}^{(i)}+\tau_{r z_{2}}^{(r)}+\tau_{r z_{2}, \bar{S}_{1}}^{(s)}+\tau_{r z_{2}, \bar{S}_{2}}^{(s)}+\tau_{r z_{2}, H}^{(s)}=0 & \text { on } \overline{\mathrm{S}}_{2} \\
\tau_{r z_{3}}^{(i)}+\tau_{r z_{3}}^{(r)}+\tau_{r z_{3}, \bar{S}_{1}}^{(s)}+\tau_{r r_{3}, \bar{S}_{2}}^{(s)}+\tau_{r r_{3}, H}^{(s)}=0 & \text { on } \mathrm{H}
\end{array} .\right.
$$

Substituting the displacement and stress expressions into Eq. (36) and then multiplying both sides of the equations by $e^{-\mathrm{i} n \theta}$ at the same time and integrating over the interval $(-\pi, \pi)$, a set of infinite algebraic equations for the unknown coefficients $A_{m}, B_{m}, C_{m}, D_{m}$ can be obtained.

\subsection{Dynamic stress concentration factor (DSCF)}

For the calculational model discussed in this paper, total stresses on the tunnel boundary $H$ can be written as Eq. (37):

$\tau_{\theta z_{3}}^{(t)}=\tau_{\theta z_{3}, \bar{S}_{1}}^{(s)}+\tau_{\theta z_{3}, \bar{S}_{2}}^{(s)}+\tau_{\theta z_{3}, H}^{(s)}+\tau_{\theta z_{3}}^{(i)}+\tau_{\theta z_{3}}^{(r)} \quad$ on $H$.

The dynamic stress concentration factor $\tau_{\theta z}^{*}$ can be defined as Eq. (38):

$\tau_{\theta z}^{*}=\left|\tau_{\theta z_{3}}^{(t)} / \tau_{0}\right|$.

In which, $\tau_{0}=\mu k W_{0}$ is the maximum amplitude of the incident stress.

\section{Calculational examples and analysis of results}

The model with the tunnel directly below the hill is used as a calculational example, indicating the influence of the existence of the canyon with different parameters on the dynamic stress concentration of the tunnel boundary, with $h$ representing the distance between $O_{1}$ and $O_{3}$. In the following, the number of incident waves is $k R_{1}=\omega R_{1} / c_{s}$, or written as $\eta=2 R_{1} / \lambda=k R_{1} / \pi$ in which $\lambda$ is the wavelength of the incident waves.

For situations with $R_{2} / R_{1}=0,0.5,1.0$ and 1.5, Fig. 3 illustrates the distribution of DSCF on the tunnel edge with different incident wave numbers and different incident angles $\alpha$ when $R_{3} / R_{1}=0.5, h / R_{1}=3.0$. Here $R_{2} / R_{1}=0$ means that the influence of the shallow buried tunnel can be ignored. When the SH wave is incident at low frequency $\eta=0.1,0.25$ and the incident angle is $\alpha=0^{\circ}, 30^{\circ}$, the existence of the canyon has different degrees of amplification on the DSCF of the tunnel. As shown in Fig. 3(e), $\eta=0.25, \alpha=0^{\circ}$, the value of $\tau_{\theta z}^{*}$ at point $\theta=0^{\circ}$ is about 4.24 in the absence of the canyon $\left(R_{2} / R_{1}=0\right)$. If the canyon is taken into account, $\tau_{\theta z}^{*}$ at the same point is magnified in three cases that $R_{2} / R_{1}=0.5,1.0$ and 1.5, and the larger the radius of the canyon, the more obvious the amplification. Compared with the state without the canyon, of 
the point increases by nearly $14 \%$ when $R_{2} / R_{1}=1.5$. With an increase of the incident angle, the effect of the canyon shows up mainly in the reduction of $\tau_{\theta z}^{*}$. When $\alpha=60^{\circ}$, $90^{\circ}$ the presence of the canyon generally decreases DSCF of the tunnel except for the case of $\eta=0.25, \alpha=60^{\circ}$ (Fig. 3(g)). It can be seen from Fig. 3(c) that the maximum value of $\tau_{\theta z}^{*}$ on the tunnel boundary is reduced by approximately $15 \%$ below the case without the canyon as $\eta=0.1$, $\alpha=60^{\circ}$, Figs. 3(i) -(q) indicate that in the case of high frequency incident of SH wave $\eta=0.75,1.25$, DSCF changes

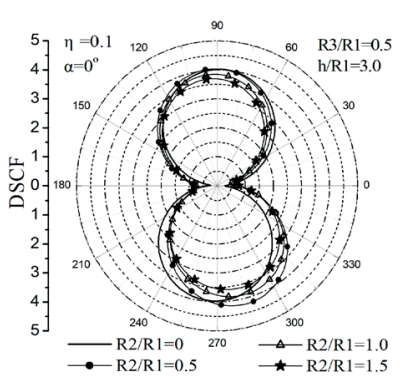

(a)

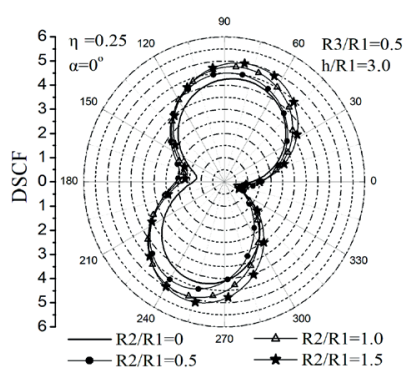

(e)

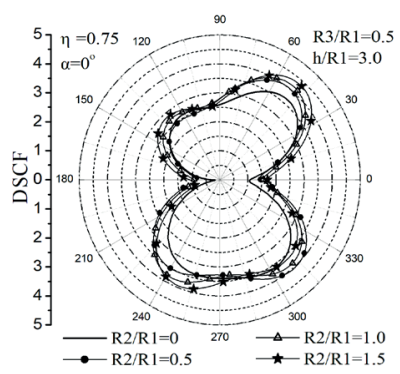

(i)

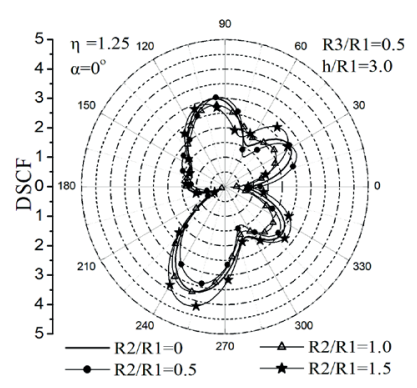

(m)

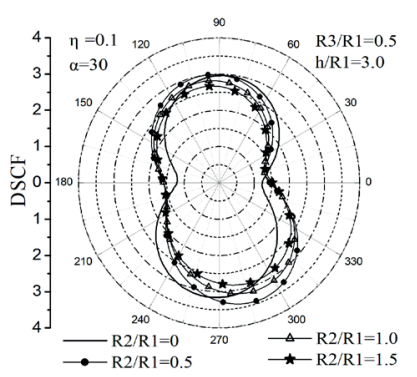

(b)

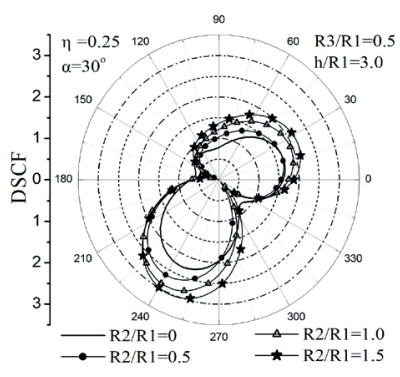

(f)

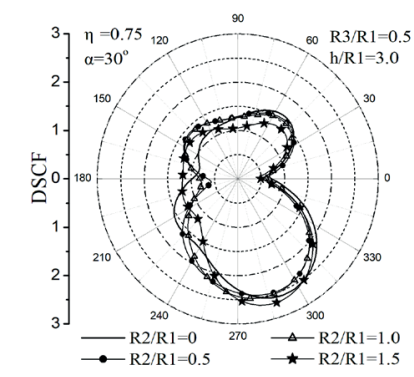

(j)

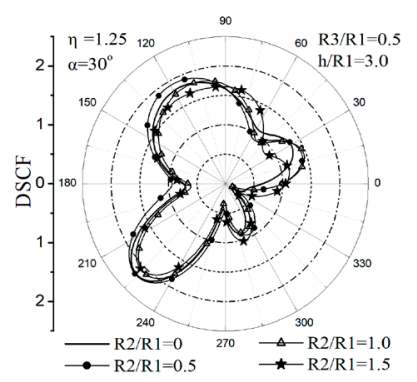

(n) more sharply on the boundary of the tunnel near the canyon side with an increase of the incident angle compared with the case without a canyon.

For different values of $R_{3} / R_{1}$, the influence of the existence and size of the canyon on the tunnel DSCF is clearly shown in Fig. 4 when $\alpha=90^{\circ}, h / R_{1}=3.0$. In general, the smaller the tunnel radius, the larger the value of $\tau_{\theta z}^{*}$ on its boundary; and as the tunnel radius increases, variation of the DSCF shows more dynamic characteristics. When other parameters are the same, the larger the radius of the

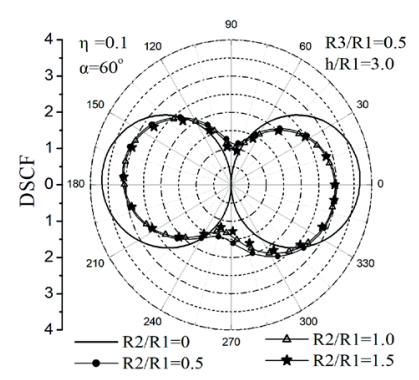

(c)

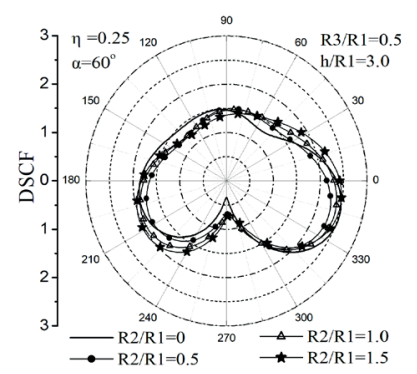

(g)

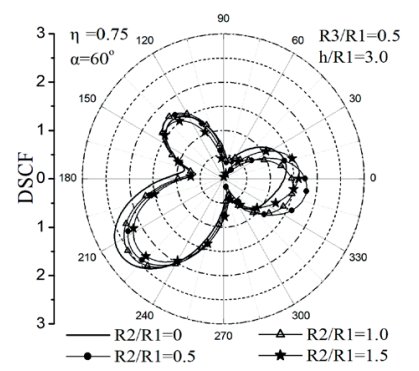

(k)

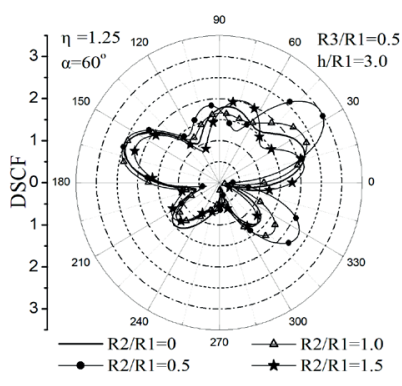

(o)

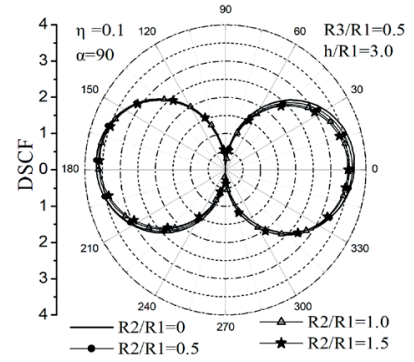

(d)

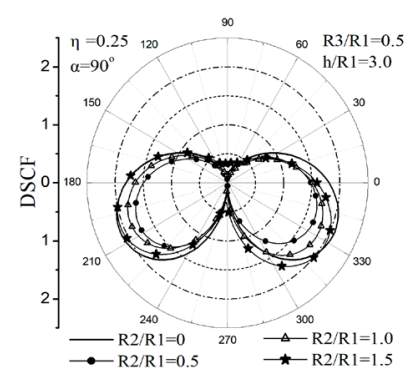

(h)

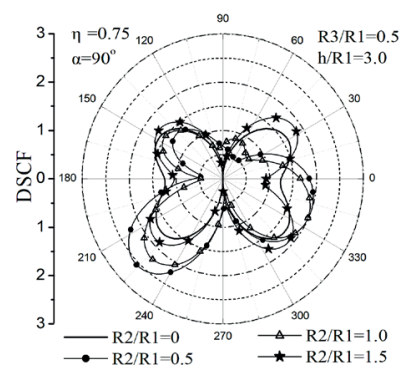

(1)

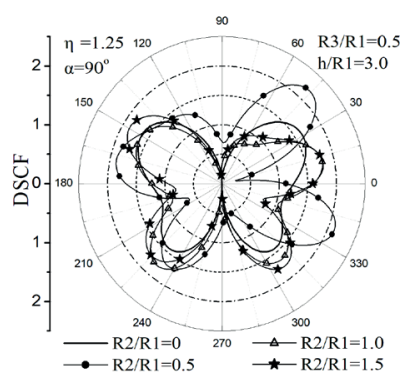

(p)

Fig. 3 Distribution of DSCF when $R_{3} / R_{1}=0.5, \mathrm{~h} / R_{1}=3.0$ 


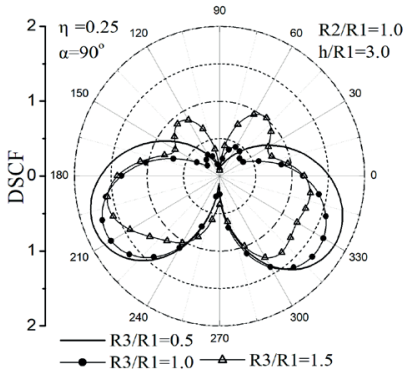

(a)

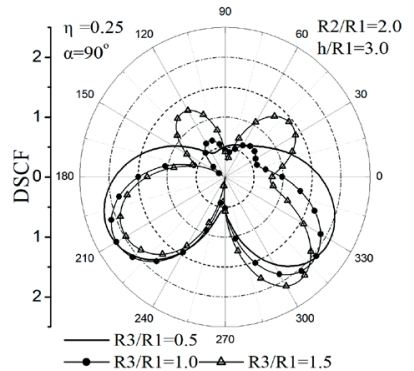

(b)

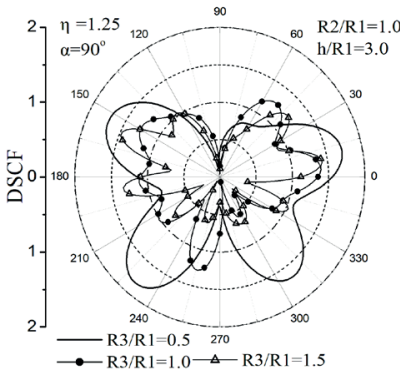

(c)

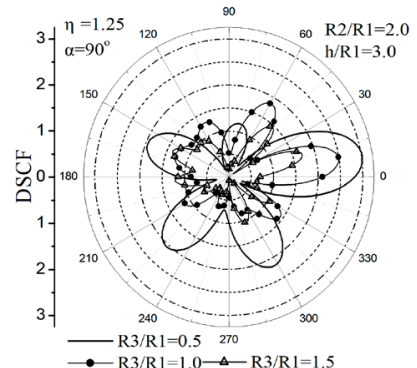

(d)

Fig. 4 Distribution of DSCF with different $R_{3} / R_{1}$ under vertical incidence of SH waves

canyon the more severe is the impact on the tunnel's DSCF, especially on the side of the tunnel nearest the canyon. For instance, $\eta=1.25, R_{3} / R_{1}=1.0$ (Figs. 4(c)-(d)), $\tau_{\theta z}^{*}$ at the point $\theta=0^{\circ}$ is about 2.1 when $R_{2} / R_{1}=2.0$, which is approximately $50 \%$ higher than in the case of $R_{2} / R_{1}=1.0$.

Fig. 5 shows the variation of $\tau_{\theta z}^{*}$ at the point $\theta=0^{\circ}$ of the tunnel with different radii of the canyon when $h / R_{1}=3.0$ and $\alpha=90^{\circ}$. It can be seen that in either case of $\eta=0.25$ or $\eta=1.25, \tau_{\theta z}^{*}$ at the point $\theta=0^{\circ}$ changes periodically with the increase of the canyon radius; and the influence of the canyon tends to be stable when $R_{2} / R_{1}>8.0$.

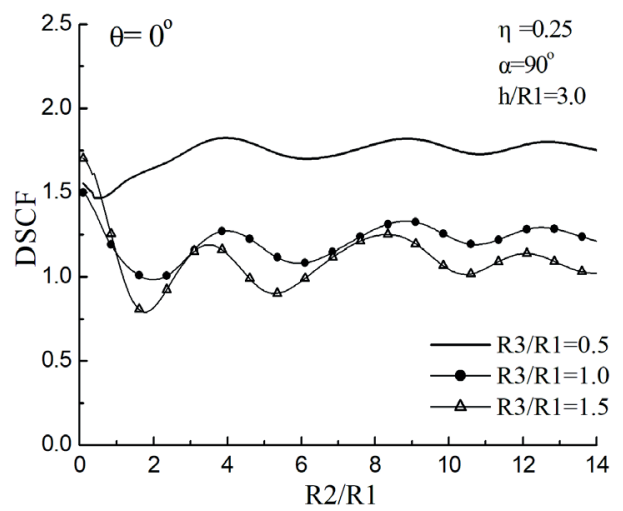

(a)

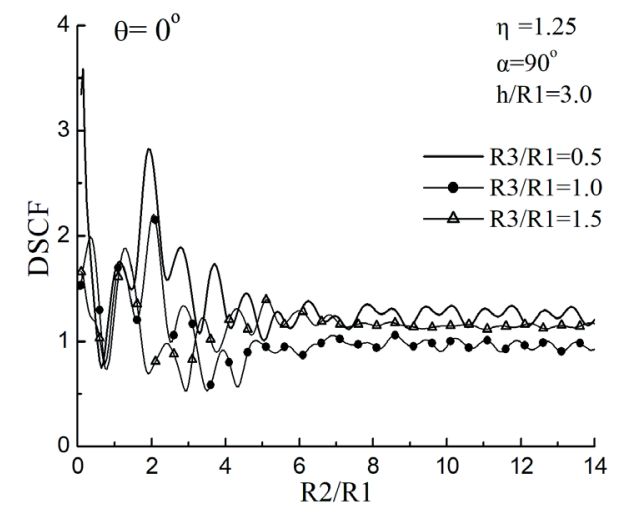

(b)

Fig. 5 Variation of $\tau_{\theta z}^{*}$ at the point $\theta=0^{\circ}$ of the tunnel with $R_{2} / R_{1}$ when $h / R_{1}=3.0$ and $\alpha=90^{\circ}$

\section{Conclusions}

The existence of the tunnel has a noticeable amplification effect on the dynamic stress of the tunnel for incident $\mathrm{SH}$ waves with low frequency and small incident angle as well as with high frequency and vertical incidence.

Under the high frequency incident SH wave, the existence of the canyon makes the dynamic effect of the tunnel near the canyon side more significant. Regardless of whether the SH wave is incident at low or high frequencies, the influence of the canyon tends to stabilize when $R_{2} / R_{1}>8.0$.

\section{Acknowledgement}

The project presented in this paper is supported by key project of university excellent young scholars for domestic and overseas visit, Anhui province (gxfxZD2016217), and development fund of Hefei University (2018ZR14ZDA).

\section{References}

[1] Lee, V. W., Trifunac, M. D. "Response of Tunnels to Incident SH-Waves", Journal of the Engineering Mechanics Division, 105(4), pp. 643-659, 1979. [online] Available at: https://www.researchgate. net/publication/255685915_Response_of_Tunnels_to_Incident_ SH_Waves [Accessed: 09 April 2019]

[2] Balendra, T., Thambiratnam, D. P., Koh, C. G., Lee, S.-L. "Dynamic response of twin circular tunnels due to incident SH-waves", Earthquake Engineering and Structural Dynamics, 12(2), pp. 181201, 1984.

https://doi.org/10.1002/eqe.4290120204

[3] Liang, J.-W., Ji, X.-D., Lee, V. W. "地下圆形祄砌隧道对沿线地震 动的影响 (I): 级数解" (Effects of an underground lined tunnel on ground motion (I): series solution), Rock and Soil Mechanics, 26(4), pp. 520-524, 2005. (in Chinese)

https://doi.org/10.16285/j.rsm.2005.04.003

[4] Liang, J. W., Ji, X. D., Lee, V. W. “地下圆形衬砌隧道对沿线地震 动的影响 (II): 数值结果” (Effects of an underground lined tunnel on ground motion (II): numerical results), Rock and Soil Mechanics, 26(5), pp. 687-692, 2005. (in Chinese) https://doi.org/10.16285/j.rsm.2005.05.003 
[5] Smerzini, C., Avilés, J., Paolucci, R., Sánchez-Sesma, F. J. "Effect of underground cavities on surface earthquake ground motion under SH wave propagation", Earthquake Engineering and Structural Dynamics, 38(12), pp. 1441-1460, 2009.

https://doi.org/10.1002/eqe.912

[6] Liang, J., Luo, H., Lee, V. W. "Diffraction of plane SH waves by a semi-circular cavity in half-space", Earthquake Science, 23(1), pp. $5-12,2010$.

https://doi.org/10.1007/s11589-009-0084-4

[7] Kara, H. F. "A note on response of tunnels to incident SH-waves near hillsides", Soil Dynamics and Earthquake Engineering, 90, pp. 138-146, 2016.

https://doi.org/10.1016/j.soildyn.2016.08.021

[8] Liu, D., Gai, B., Tao, G. "Applications of the method of complex functions to dynamic stress concentration", Wave Motion, 4(3), pp. 293-304, 1982.

https://doi.org/10.1016/0165-2125(82)90025-7

[9] Liu, D. "Dynamic stress concentration around a circular hole due to SH-wave in anisotropic media", Acta Mechanica Sinica, 4(2), pp. $146-155,1988$.

https://doi.org/10.1007/bf02487716

[10] Wang, Y., Liu, D. "SH波入射时浅埋衬砌结构的动力分析" (Dynamic Analysis for Shallow-embedded Lining Structure Impacted by Incident SH-wave), Journal of Harbin Engineering University, 23(6), pp. 43-47, 2002. (in Chinese)

https://doi.org/10.3969/j.issn.1006-7043.2002.06.012

[11] Liu, D. K., Lin, H. "浅埋的圆柱形孔洞对 SH波的散射与地震动" (Scattering of SH-waves by a shallow buried cylindrical cavity and the ground motion), Explosion and Shock Waves, 23(1), pp. 6-12, 2003. (in Chinese)

https://doi.org/10.3321/j.issn:1001-1455.2003.01.002

[12] Chen, Z. G. "浅埋孔洞对地表反平面运动的影响" (Effects of shallow buried cavity on anti-plane ground motion), Rock and Soil Mechanics, 28(8), pp. 1655-1660, 2007. (in Chinese) https://doi.org/10.3969/j.issn.1000-7598.2007.08.023

[13] Parvanova, S. L., Dineva, P. S., Manolis, G. D., Frank, W. "Seismic response of lined tunnels in the half-plane with surface topography", Bulletin of Earthquake Engineering, 12(2), pp. 981-1005, 2014. https://doi.org/10.1007/s10518-013-9546-0
[14] Li, P., Song, E.-X. "Three-dimensional numerical analysis for the longitudinal seismic response of tunnels under an asynchronous wave input", Computers and Geotechnics, 63, pp. 229-243, 2015. https://doi.org/10.1016/j.compgeo.2014.10.003

[15] Fu, J., Liang, J. "Dynamic soil-tunnel interaction in layered halfspace for incident plane SH waves", Earthquake Engineering and Engineering Vibration, 15(4), pp. 715-727, 2016. https://doi.org/10.1007/s11803-016-0360-y

[16] Lee, V. W., Chen, S., Hsu, I. R. "Antiplane Diffraction from Canyon Above Subsurface Unlined Tunnel", Journal of Engineering Mechanics, 125(6), pp. 668-675, 1999. https://doi.org/10.1061/(asce)0733-9399(1999)125:6(668)

[17] Lee, V. W., Manoogian, M. E., Chen, S. "Antiplane SH-deformations near a surface rigid foundation above a subsurface rigid circular tunnel", Earthquake Engineering and Engineering Vibration, 1(1), pp. 27-35, 2002. https://doi.org/10.1007/s11803-002-0004-2

[18] Lee, V. W., Luo, H., Liang, J. "Diffraction of anti-plane SH waves by a semi-circular cylindrical hill with an inside concentric semicircular tunnel", Earthquake Engineering and Engineering Vibration, 3(2), pp. 249-262, 2004.

https://doi.org/10.1007/bf02858239

[19] Liu, D. K., Wang, G. Q. "浅埋圆形孔洞附近的半圆形凸起对 SH 波的散射" (Antiplane SH-Deformation of a Semi-Cylindrical Hill above a Subsurface Cavity), Chinese Journal of Theoretical and Applied Mechanics, 38(2), pp. 109-128, 2006. (in Chinese) https://doi.org/10.3321/j.issn:0459-1879.2006.02.009

[20] Lv, X. T. "Scattering of SH-wave by multiple semi-cylindrical hills above a subsurface elastic cylindrical inclusion", Journal of Vibroengineering, 16(6), pp. 2695-2701, 2014. [online] Available at: https://www.jvejournals.com/article/15055 [Accessed:09April 2019]

[21] Amornwongpaibun, A., Luo, H., Lee, V.W. "Scattering of Anti-Plane (SH) Waves by a Shallow Semi-Elliptical Hill with a Concentric Elliptical Tunnel", Journal of Earthquake Engineering, 20(3), pp. 363-382, 2016.

https://doi.org/10.1080/13632469.2015.1085465

[22] Alielahi, H., Kamalian, M., Adampira, M. "A BEM investigation on the influence of underground cavities on the seismic response of canyons", Acta Geotechnica, 11(2), pp. 391-413, 2016. https://doi.org/10.1007/s11440-015-0387-7 